\title{
ClonorESTdb: a comprehensive database for Clonorchis sinensis EST sequences
}

\author{
Dae-Won Kim ${ }^{\dagger}$, Won Gi Yoo ${ }^{\dagger}$, Sanghyun Lee, Myoung-Ro Lee, Yu-Jung Kim, Shin-Hyeong Cho, Won-Ja Lee \\ and Jung-Won Ju*
}

\begin{abstract}
Background: Clonorchiasis, which is primarily caused by liver fluke (Platyhelminthes), is a fatal infectious disease that is mainly associated with bile duct malignancy and the subsequent development of cholangiocarcinoma. Thus, a genomic approach now represents an important step to further our knowledge of biology and the pathology of these parasites. The results of expressed sequence tags (ESTs) sequencing need to be well organized into databases to provide an integrated set of tools and functional information.

Findings: Here, the ClonorESTdb database represents a collection of Clonorchis sinensis ESTs that is intended as a resource for parasite functional genomics. A total of 55,736 successful EST sequences, which are cleaned and clustered into non-redundant 13,305 C. sinensis assembled EST sequences (6,497 clusters and 6,808 singletons), were obtained from three in-house prepared cDNA libraries of $C$. sinensis at different developmental stages. The assembled consensus sequences were annotated using the BLAST algorithm or/and hmm against NCBI NR, UniProt, KEGG and InterProScan. The ClonorESTdb database provides functional annotation, their expression profiles, tandem repeats and putative single nucleotide polymorphisms with utility tools such as local BLAST search and text retrieval.
\end{abstract}

Conclusions: This resource enables the researcher to identify and compare expression signatures under different biological stages and promotes ongoing parasite drug and vaccine development and biological research.

Database URL: http://pathod.cdc.go.kr/clonorestdb/

Keywords: Clonorchis sinensis, Expressed sequence tags (ESTs), Transcriptome, Database

\section{Findings}

Clonorchis sinensis is the human liver fluke of the class Trematoda (phylum Platyhelminthes: Digenea). The human host is infected by consuming raw and inadequately cooked freshwater fish with $C$. sinensis metacercariae. Clonorchiasis is a common infectious disease in many eastern Asian countries, including Korea, China, Japan and Vietnam. It is estimated that Clonorchiasis affects approximately 35 million people worldwide [1] and more than 600 million people are at risk of infection in East Asia and Eastern Europe [2].

Metacercariae of $C$. sinensis exist in the small intestine and the juvenile worms migrate up through the Ampulla of

\footnotetext{
*Correspondence: leewonja@gmail.com; junomics@gmail.com ${ }^{\dagger}$ Equal contributors

Division of Malaria and Parasitic Diseases, Centre for Immunology and Pathology, Korea National Institute of Health, Chungbuk 363-951, Republic of
} Korea

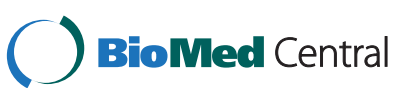

(c) 2014 Kim et al.; licensee BioMed Central Ltd. This is an Open Access article distributed under the terms of the Creative Commons Attribution License (http://creativecommons.org/licenses/by/4.0), which permits unrestricted use, distribution, and reproduction in any medium, provided the original work is properly credited. The Creative Commons Public Domain Dedication waiver (http://creativecommons.org/publicdomain/zero/1.0/) applies to the data made available in this article unless otherwise stated.
Vater and the common bile duct [3]. The livers of patients with clonorchiasis appear almost normal in cases of light infections, but slightly dilated and thickened peripheral bile ducts are present in cases of heavy infections. Patients with clonorchiasis persistently suffer from fatigue, jaundice, abdominal distress and indigestion [4]. Chronic infection can cause several hepatobiliary disease manifestations, such as cholangitis, cholecystitis, cholelithiasis, hepatomegaly and fibrosis of the periportal tract [5-7]. Although C. sinensis is officially recognized as a biological human group I carcinogen by the International Agency for Research on Cancer (IARC) and the World Health Organization (WHO) [8], the molecular and cellular biology of $C$. sinensis has been significantly underexplored due to the lack of genomic database resources from well-isolated full-length cDNAs.

The advent of next-generation sequencing machines that involve GS 454 [9], Solexa [10] and SOLiD [11] are revolutionizing molecular biology by generating hundreds 
of thousands of sequencing reads in parallel. The genome and transcriptome sequences of a growing number of model organisms have been published in recent years, which have drawn new insights into parasite research $[12,13]$. However, the de novo large-scale sequencing of a non-model parasite is still a laborious task and an interesting challenge. Expressed sequence tags (ESTs) are a cost effective alternative and a powerful tool that provides sufficient information about functional proteins. In particular, the ESTs from a full-length cDNA library allow researchers to be cloned and provide material sources so that many intriguing biological issues can be isolated.

The aim of this study is to provide important database resources for the characterization and understanding of the functional genes of $C$. sinensis. Here, we have constructed and described ClonorESTdb, a web-based ESTs database resource that involves systematic functional annotation, which comprises more than 55,736 high-quality ESTs based on three full-length enriched cDNA libraries. The ESTs obtained were assembled into 13,305 C. sinensis Assembled EST sequences (CsAEs) comprising 6,497 clusters and 6,808 singletons by aligning CsAEs onto the non-redundant public NCBI NR database, UniProt, KEGG, InterProScan and Gene Ontology (GO). The ClonorESTdb database described here provides key insights into the differential gene expression of $C$. sinensis in a range of developmentally relevant conditions.

\section{Database architecture}

The ClonorESTdb database runs on a RedHat Enterprise Linux 5.5 platform with the Apache web server version 2.2. We also used the relational Oracle database $11 \mathrm{~g}$ standard version to develop and support an integrated database schema for storing sequence data, preprocessed data and final functional annotation. The web application was implemented with JSP (Java Server Pages), JavaServelet technology and the AJAX framework. The web interfaces were designed using HTML language with some scripts in JavaScript and the pages utilized cascading style sheet (CSS) properties. The database is currently optimized to work best with Microsoft Internet Explorer 8 (optimal resolution $1024 \times 800)$.

\section{Data source}

We constructed full-length cDNA libraries (adult, metacercaria and egg) from C. sinensis and generated large-scale 60,768 ESTs data by 5 '-end sequencing of individual clones [14]. All of the raw and cleaned data can be downloaded from the ClonorESTdb database.

\section{The pipeline for constructing the database}

In our study, a total of 55,736 C. sinensis EST sequences that were derived from three cDNA libraries (adult, metacercaria and egg) were used to construct the database.
To analyze the data, we developed a pipeline for the ClonorEST Project divided into three steps: sequence cleaning, sequence clustering and assembly, and automatic annotation.

\section{Sequence cleaning}

Cleaning is an important part of processing and is used to obtain high-quality EST datasets from raw EST sequences. After base calling was performed using Phred [15], the cleaning process implemented Cross_match (version 0.990329) for masking any vector and contaminant sequences, SeqClean (http://seqclean.sourceforge.net/) for eliminating undetermined bases, poly $(\mathrm{A})$ tails and low complexity elements, RepeatMasker (http://www. repeatmasker.org/) for removing interspersed repeats, such as SINEs (short interspersed nuclear element), LINEs (long interspersed nuclear elements), LTRs (long terminal repeat) and DNA elements included in the Repbase repetitive element library (http://www.girinst. org/) [16] (Figure 1A).

\section{Sequence clustering and assembly}

The clustering procedure was a basic step that was used to collect overlapping CsAEs sequences that originated from the same transcript of a single gene; this is performed to reduce redundancy. The assembly procedure is executed to align and merge many overlapping EST sequences of a much longer DNA sequence to reconstruct a putative fulllength transcript sequence. For clustering and assembling, we used TGICL to create a grouping EST sequences and CAP3 for assembling the clustered EST sequences $[17,18]$ (Figure 1B).

\section{Automatic functional annotation}

For more accuracy and further variety of functional annotation, we used various annotation algorithms and public databases. First, we assigned putative functions to the CsAEs based on BLASTN (Query Coverage $\geq 80.0 \%$, Identity $\geq 70.0 \%$, E-value $\leq 1.0 \mathrm{e}-5)$, BLASTX and TBLASTX (Match No. $\geq 30$ aa, Identity $\geq 25.0 \%$, E-value $\leq 1.0 \mathrm{e}-5$ ) searches against the GenBank NT and NR databases (ftp://ftp.ncbi.nih.gov/blast/db/FASTA/). Second, metabolic pathways are extremely important for correctly inferring pathogen invasion, host defense, adaptation, pathogen life cycle and host-pathogen interactions. To get a rationale for the development of anti-parasitic drugs and vaccines, we must identify all parasite-specific metabolic pathways. To identify the pathways, additional annotations were created against the UniProtKB database (http://www.ebi.ac.uk/ uniprot) and the KEGG database (http://www.genome. jp) using BLASTX (Match No. $\geq 30$ aa, Identity $\geq 25.0 \%$, E-value $\leq 1.0 \mathrm{e}-5)$. All BLAST algorithms were implemented using a TimeLogic DeCypher system (Active Motif, Inc., http://www.activemotif.com). We also used the 


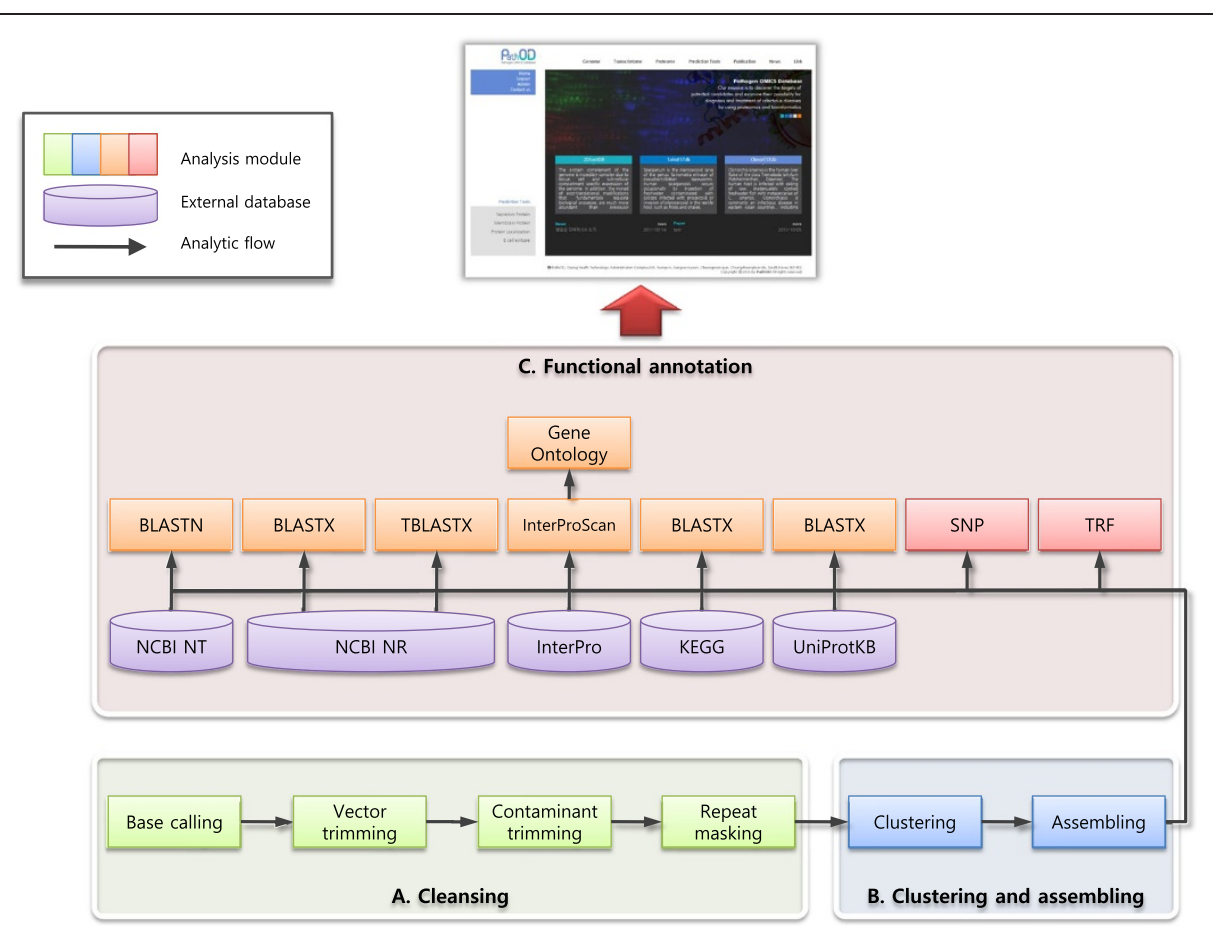

Figure 1 Workflow scheme of an EST analytic process. A. The cleaning step is composed of base calling (Phred), vector masking (cross_match), contaminant trimming (SeqClean) and repeat masking (RepeatMasker). B. The cleaned EST sequences are sent to the next step: clustering and assembling (TGICL). C. CSAEs sequences are aligned with various public databases for assigning their putative function and predicting structural variation (AutoSNP and TRF).

InterProScan tool to extract additional functional domains (E-value $\leq 1.0 \mathrm{e}-4)$. To gain a better classification of the biological function of the CsAEs, an analysis of the functions was conducted using GO terms according to three categories: molecular function, biological process and cellular component. We used Tandem Repeats Finder (TRF) and AutoSNP to detect structural variations $[19,20]$ (Figure 1C).

\section{Utility and discussion}

The aims of this database project included 1) the construction of an integrative database of the C. sinensis transcriptome, 2) the maintenance of a well-organized functional annotation and the identification and characterization of factors such as parasite specific antigen and 3) data-mining for tissue-specific expression to discover key pathways related to parasite proliferation that are essential to its maintenance. The database presented here and named ClonorESTdb consists of 55,736 EST entries from $C$. sinensis at three stages including adult, metacercaria and egg.

\section{ClonorESTdb web interface}

The ClonorESTdb provides a user-friendly interface with the seven following options in the main menu: (1) a detailed pre-processing report, (2) clustering and assembling report and viewer, (3) various functional annotation report and (4) download all of the raw data and the analyzed result. The data can be viewed at various degrees of detail, either as an overview (a list of search results) or as a detailed results page for a selected sequence and functional annotation.

\section{Pre-processing and assembling report}

After pre-processing, all of results are stored in a database for evaluation and reporting, according to four steps (base calling, vector trimming, contamination, trimming and repeat masking). The pre-processing shows a summary table of the pre-processing steps. The assembly report of ClonorESTdb provides a comprehensive summary of the status of contigs and singletons, such as sequencing status and clustering information. Furthermore, each sequence and each contig were assigned a detailed on-the-fly page where the ID of the nucleotide sequence can be clicked. In each contig page, the graphical display of the contigs and the contig alignment sequence information are provided. We also provide the module of a trace viewer that allows users to evaluate the result of the sequencing of raw data that was derived from the machine on the web (Figure 2A).

\section{Annotation report}

For various functional annotation reporting, the online database of ClonorESTdb provides a comprehensive 


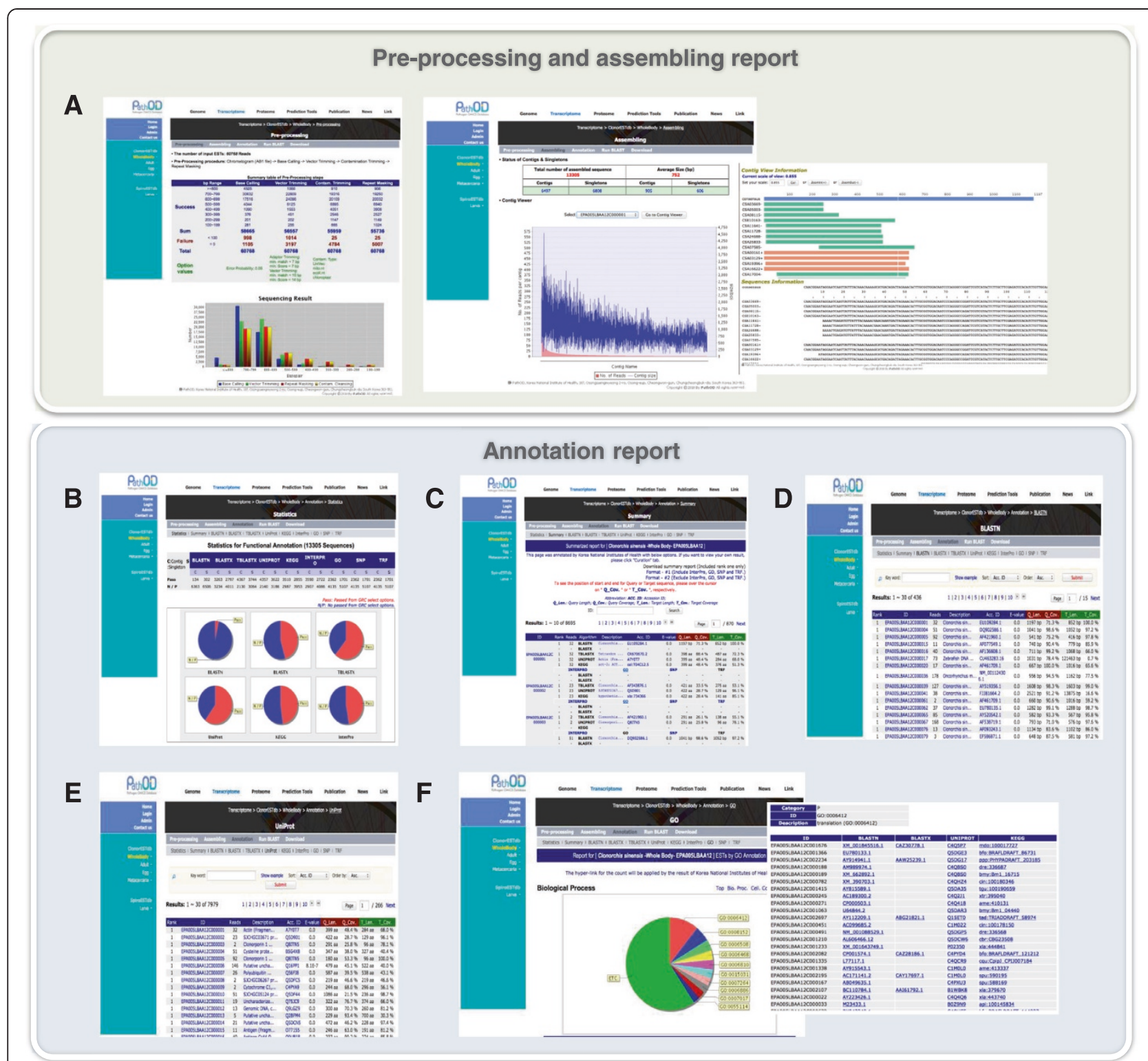

Figure 2 Snapshots of the ClonorESTdb web application. The panels show examples for various general functions. A. An example of a pre-processing report, which shows the cleaning sequences, assembly status, contig view and trace view. B. A statistics page showing an annotation result. C. A summary page showing various annotation results. D. A BLASTX annotation report page. E. A UniProt annotation report page. F. A GO annotation report page.

information system for analyzing data from the 55,736 EST sequencing project. An annotation report provides 12 categories of analysis information to allow users to easily monitor all of the analyzed EST datasets. The "statistics" page provides a numeric table of functional annotation and the annotated species distribution derived from all the algorithms (Figure 2B). On the "summary report" page, users can see all of the detailed annotation information regarding how the CsAEs were annotated in the system and the users can download entire annotation files as Excel files (Figure 2C). The annotation results against various databases contain clone and contig information, including the sequence, putative identification, annotation detailed information, a link to the nearest homologue in the public database, the length of the homologous sequence and the percentage identity of the nearest match. In addition, existing information for all CsAEs in an EST set can be retrieved using full-text matching (against read id, consensus id, gene name, gene accession number, functional description and E-value score) from the search tool (Figure 2D). In this section, ClonorESTdb provides access to the enzyme and pathway information in UniProt and KEGG linked to an external database. Users can mine the enzyme information from the 
annotated results of KEGG and UniProt databases that contained an EC number in the description (Figure 2E). Here, for the task of representing and processing information by domain analysis in the "InterPro" page, their products and their functions are presented in GO categories (Figure 2F). When working on one or more given individuals or species, the biologist may wish to search for markers in intra-specific polymorphisms or intra-specific polymorphisms. Thus, we also added single nucleotide polymorphisms (SNPs) and simple tandem repeats (STRs) data.

\section{Conclusion}

In summary, ClonorESTdb is the first incorporated online transcriptome database of $C$. sinensis that can be freely accessed and downloaded. Moreover, this database provides various utility functions for similarity searches, transcriptome statistic information and investigation of user-supplied genomics and transcriptome sequences. The increasing amounts of genomics data that are derived from the advent of high-throughput technology will further stimulate the integration of ClonorESTdb. Studies of this project are undoubtedly of notable interest to many biologists given the complex genetic, biochemical, physiological and evolutionary processes that remain at the heart of host-pathogen interactions. In addition, we will continue to collect known $C$. sinensis full-length cDNAs to update the database for public use. The annotated functional protein presented herein and our knowledge database will be useful for parasite researchers who wish to clone and confirm full-length $C$. sinensis cDNAs of interest.

\section{Availability}

The ClonorESTdb is open and freely available. All questions, comments and requests should be sent via email to todaewon@gmail.com.

Project name: ClonorESTdb.

Project home page: http://pathod.cdc.go.kr/clonorestdb/. Operating system: Linux.

Programming languages: HTML, JSP, CSS3, JavaScript, AJAX, Oracle.

Other requirements: none.

License: None required.

\section{Competing interests}

The authors declare that they have no competing interests.

\section{Authors' contributions}

DK and WY designed and implemented the database and website and wrote the manuscript, and DK, WY and SL developed the web interfaces, assisted with the design of the database and performed database system administration. SC, YK and ML helped with the preparation of the EST data sets (sample collection, CDNA library construction and sequencing). WL and $\mathrm{JJ}$ served as the principal investigators of the project. All authors contributed to the writing of the manuscript and have read and approved the final submitted version.

\section{Acknowledgements}

The authors thank the pathogen research community at the Korea National Institutes of Health for valuable input on this project and suggestions for building and maintaining this database.

\section{Funding}

This work was supported by the Pathogenic Proteome Management Program (4800-4847-300) from the Korea National Institute of Health, Korea Centers for Disease Control and Prevention.

Received: 13 March 2014 Accepted: 19 June 2014

Published: 24 June 2014

\section{References}

1. Lun ZR, Gasser RB, Lai DH, Li AX, Zhu XQ, Yu XB, Fang YY: Clonorchiasis: a key foodborne zoonosis in China. Lancet Infect Dis 2005, 5:31-41.

2. Keiser J, Utzinger J: Emerging foodborne trematodiasis. Emerg Infect Dis 2005, 11:1507-1514

3. Kaewkes S: Taxonomy and biology of liver flukes. Acta Trop 2003, 88:177-186.

4. Kim TI, Na BK, Hong SJ: Functional genes and proteins of Clonorchis sinensis. Korean J Parasitol 2009, 47(Suppl):S59-S68.

5. Sithithaworn P, Haswell-Elkins MR, Mairiang P, Satarug S, Mairiang E, Vatanasapt V. Elkins DB: Parasite-associated morbidity: liver fluke infection and bile duct cancer in northeast Thailand. Int J Parasitol 1994, 24:833-843.

6. Marcos LA, Terashima A, Gotuzzo E: Update on hepatobiliary flukes: fascioliasis, opisthorchiasis and clonorchiasis. Curr Opin Infect Dis 2008, 21:523-530

7. Lim JH: Radiologic findings of clonorchiasis. AJR Am J Roentgenol 1990, 155:1001-1008.

8. Bouvard V, Baan R, Straif K, Grosse Y, Secretan B, El Ghissassi F, Benbrahim-Tallaa L, Guha N, Freeman C, Galichet L, Cogliano V, WHO International Agency for Research on Cancer Monograph Working Group: A review of human carcinogens-Part B: biological agents. Lancet Oncol 2009, 10:321-322.

9. Margulies M, Egholm M, Altman WE, Attiya S, Bader JS, Bemben LA, Berka J, Braverman MS, Chen YJ, Chen Z, Dewell SB, Du L, Fierro JM, Gomes XV, Godwin BC, He W, Helgesen S, Ho CH, Irzyk GP, Jando SC, Alenquer ML, Jarvie TP, Jirage KB, Kim JB, Knight JR, Lanza JR, Leamon JH, Lefkowitz SM, Lei M, Li J, et al: Genome sequencing in microfabricated high-density picolitre reactors. Nature 2005, 437:376-380

10. Bentley DR, Balasubramanian S, Swerdlow HP, Smith GP, Milton J, Brown CG, Hall KP, Evers DJ, Barnes CL, Bignell HR, Boutell JM, Bryant J, Carter RJ, Keira Cheetham R, Cox AJ, Ellis DJ, Flatbush MR, Gormley NA, Humphray SJ, Irving LJ, Karbelashvili MS, Kirk SM, Li H, Liu X, Maisinger KS, Murray L, Obradovic B, Ost T, Parkinson ML, Pratt MR, et al: Accurate whole human genome sequencing using reversible terminator chemistry. Nature 2008 456:53-59.

11. Shendure J, Porreca GJ, Reppas NB, Lin X, McCutcheon JP, Rosenbaum AM, Wang MD, Zhang K, Mitra RD, Church GM: Accurate multiplex polony sequencing of an evolved bacterial genome. Science 2005, 309:1728-1732.

12. Berriman M, Haas BJ, Loverde PT, Wilson RA, Dillon GP, Cerqueira GC, Mashiyama ST, Al-Lazikani B, Andrade LF, Ashton PD, Aslett MA, Bartholomeu DC, Blandin G, Caffrey CR, Coghlan A, Coulson R, Day TA, Delcher A, DeMarco R, Djikeng A, Eyre T, Gamble JA, Ghedin E, Gu Y, Hertz-Fowler C, Hirai H, Hirai Y, Houston R, Ivens A, Johnston DA: The genome of the blood fluke Schistosoma mansoni. Nature 2009, 460:352-358.

13. Zhou Y, Zheng H, Chen Y, Zhang L, Wang K, Guo J, Huang Z, Zhang B, Huang W, Jin K, Dou T, Hasegawa M, Wang L, Zhang Y, Zhou J, Tao L, Cao Z, Li Y, Vinar T, Brejova B, Brown D, Li M, Miller DJ, Blair D, Zhong Y, Chen Z, Liu F, Hu W, Wang ZQ, Zhang QH, et al: The Schistosoma japonicum genome reveals features of host-parasite interplay. Nature 2009, 460:345-351.

14. Yoo WG, Kim DW, Ju JW, Cho PY, Kim Tl, Cho SH, Choi SH, Park HS, Kim TS, Hong SJ: Developmental transcriptomic features of the carcinogenic liver fluke, Clonorchis sinensis. PLoS Negl Trop Dis 2011, 5:e1208.

15. Ewing B, Hillier L, Wendl MC, Green P: Base-calling of automated sequencer traces using phred. I. Accuracy assessment. Genome Res 1998, 8:175-185.

16. Jurka J, Kapitonov W, Pavlicek A, Klonowski P, Kohany O, Walichiewicz J: Repbase Update, a database of eukaryotic repetitive elements. Cytogenet Genome Res 2005, 110:462-467.

17. Pertea G, Huang $X$, Liang F, Antonescu V, Sultana R, Karamycheva S, Lee $Y$, White J, Cheung F, Parvizi B, Tsai J, Quackenbush J: TIGR Gene Indices clustering tools (TGICL): a software system for fast clustering of large EST datasets. Bioinformatics 2003, 19:651-652. 
18. Huang X, Madan A: CAP3: A DNA sequence assembly program. Genome Res 1999, 9:868-877.

19. Benson G: Tandem repeats finder: a program to analyze DNA sequences. Nucleic Acids Res 1999, 27:573-580.

20. Barker G, Batley J, O' Sullivan H, Edwards KJ, Edwards D: Redundancy based detection of sequence polymorphisms in expressed sequence tag data using autoSNP. Bioinformatics 2003, 19:421-422.

doi:10.1186/1756-0500-7-388

Cite this article as: Kim et al: ClonorESTdb: a comprehensive database

for Clonorchis sinensis EST sequences. BMC Research Notes 2014 7:388.

\section{Submit your next manuscript to BioMed Central and take full advantage of:}

- Convenient online submission

- Thorough peer review

- No space constraints or color figure charges

- Immediate publication on acceptance

- Inclusion in PubMed, CAS, Scopus and Google Scholar

- Research which is freely available for redistribution 